\title{
45,X Turner syndrome with normal ovarial function and multiple malformations of the aorta
}

\author{
T.D. Apostolopoulos, M.K. Kyriakidis, S.A. Kitsiou', A.D. \\ Galla-Voumvouraki', A.N. Tsezou' and P.K. Toutouzas
}

Cardiological Department, Hippocration Hospital, University of Athens and ${ }^{1}$ Second Department of Pediatrics, Gene:ic Unit, 'P amd A Kyriakou' Hospital, University of Athens, Greece

\begin{abstract}
Summary: We present a case of a female patient with monosomy of $\mathbf{X}$ chromosome in peripheral lymphocytes and skin fibroblasts, normal ovarian function and associated multiple congenital abnormalities of the aorta: bicuspid aortic valve, dilatation of the ascending aorta and multiple cystic structures of the aortic wall, complicated by endarteritis. We review the literature on fertile women with $45, X$ karyotype and the possible pathogenetic mechanisms of the aortic defects described as 'cystic medial necrosis of the aorta'.
\end{abstract}

\section{Introduction}

Sporadic cases of fertile women with 45,X Turner syndrome have been reported,' most of them diagnosed cytogenetically after the investigation for secondary amenorhea and short stature. Turner syndrome could be presumed in the presence of normal ovarian function in women with typical morphological and cardiovascular anomalies. ${ }^{2-5}$

\section{Case report}

Our patient is a 36 year old woman who had experienced menarche at 12 years of age. Her periods had been regular (29 days apart) and usually lasted for 4 days. At age 24 she had an induced abortion. Her stature was short $(147 \mathrm{~cm})$ and she presented several stigmata of Turner's gonadal dysgenesis, that is, blepharoptosis, antimongoloid slant of the palpebral fissures, retruded mandible, short broad neck, pterygium coli, shield chest, cubitus valgus, clinodactyly $\mathrm{V}$ and short IV metacarpals. Her breasts were well developed and the secondary sex characteristics were normal.

She was admitted to our hospital at age 36 with acute anterior chest pain, vomiting and a low grade fever, which on the third day exceeded $39^{\circ} \mathrm{C}$. She had sinus tachycardia, a systolic murmur over the aortic area and left pleural effusion (exudative, with inflammatory and reactive mesothelial cells, and negative cultures). Except for mild anaemia, a

Correspondence: T. Apostolopoulos, M.D., 9 Kountouriotou Street, Pefki, Athens 151 21, Greece. Accepted: 5 July 1993 raised ESR and leucocytosis, the laboratory investigation for fever of unknown origin was negative.

Transthoracic and transoesophageal echocatdiography demonstrated bicuspid aortic valve wiôn $\overrightarrow{0}$ mild stenosis, dilatation of the ascending aorta, intraluminal structure at the aortic arch movim with the cardiac cycles and septum-like structures at the anterior wall of the descending aorta (Figure 1). To exclude the possibility of dissection, an aortography was performed, which revealed $a \stackrel{\circ}{\mathbb{Q}}$ vague filling defect at the base of the aortic arch, as well as other small even filling defects in theo descending aorta (Figure 2). Magnetic resonance imaging (Figure 3) established marked thickening of the wall of the base of the aortic arch and an intraluminal structure, which originated from this윽 mural thickening. Also, multiple cystic structuresof the anterior wall of the descending aorta were 3 . found equivalent to the filling defects of the aortography. With the possible diagnosis of 3 bacterial endarteritis of the aortic arch, she was given therapy with penicillin $\mathrm{G}$ plus gentamicin and had an excellent clinical course.

\section{Cytogenetic investigation}

Cytogenetic analysis was undertaken on peripheral ${ }_{O}^{\mathrm{C}}$ blood and skin. Chromosomal banding was obtained by the GTG method. A total of $200^{\circ}$ metaphases from the standard lymphocyte culture (PHA, 72 hours) were studied and another 80 from $_{\square}^{-}$ the fibroblast culture. The karyotype identified was $45, X$. On buccal smear, X-chromatin negative nuclei were revealed. 

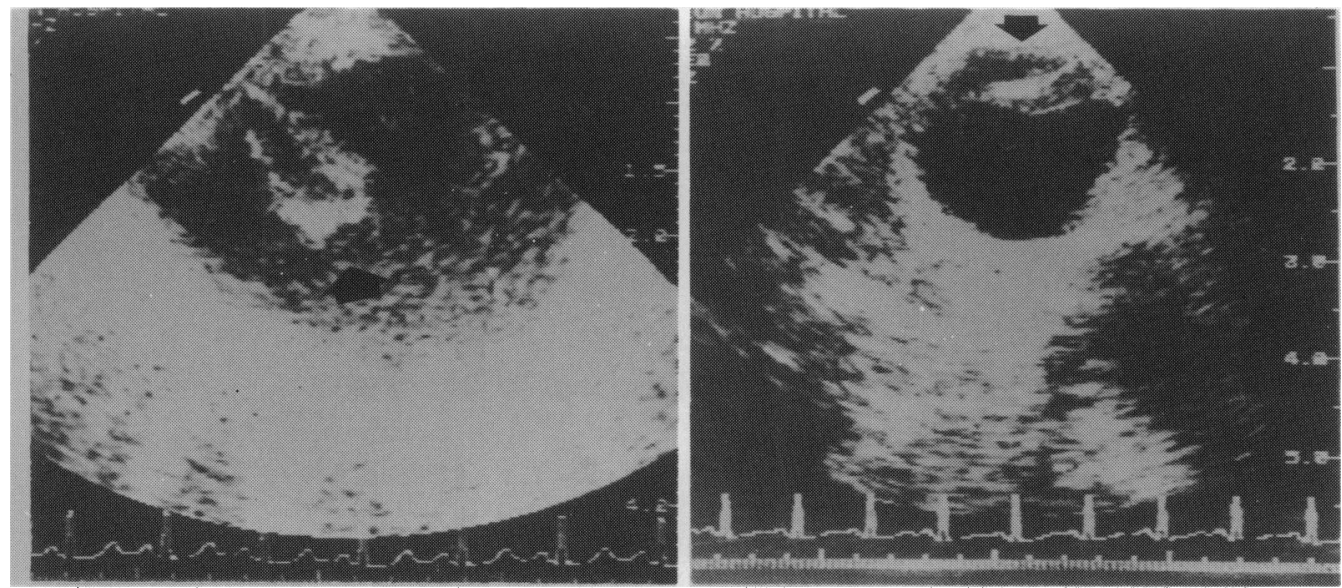

Figure 1 Transoesophageal echo of the patient showing the intraluminal structure of the aortic arch and septum-like structures of the descending aorta.
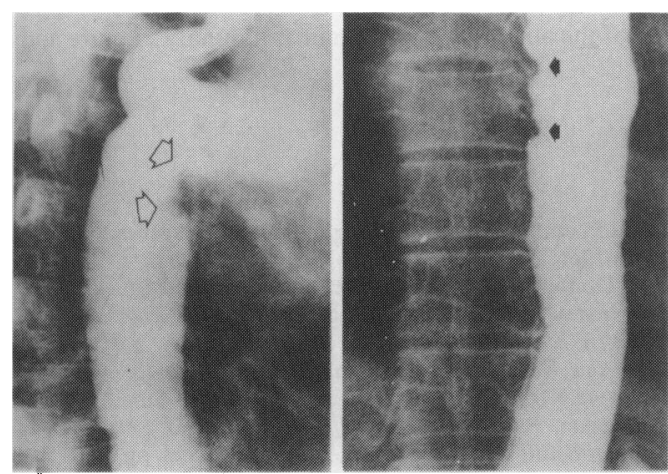

Figure 2 Aortography of the patient showing the filling defects of the aortic arch and the descending aorta.

\section{Discussion}

Our patient with 45,X Turner phenotype has normal ovarian function and a history of pregnancy. Thirteen fertile females with $45, X$ karyotype and a greater number of cases with $45, X / 46, X X$ or $45, \mathrm{X} / 47, \mathrm{XXX}$ mosaicism have been previously reported. ${ }^{1}$ A possible suggestion is that among $45, X$ women entering reproductive age, there is a percentage with some preservation of ovarian follicular activity and menstrual function. ${ }^{6,7}$ The possibility of an ovary, or a segment thereof, harbouring cells which have the normal female chromosome complement in women with $45, X$ Turner phenotype, is another explanation given by
Shokeir. ${ }^{8}$ In our patient the ultrasound investigation revealed normal ovaries and functioning follicles in spite of the consistent finding of monosomic X population of cells in both lymphocytes and fibroblasts. Such women might never have been cytogenetically diagnosed unless they experience secondary amenorrhea with symptoms of premature menopause or life-threatening cardiovascular complications, as in our patient.

Increased incidence of aortic dissection has been observed in Turner syndrome, usually complicating other congenital malformations as aortic coarctation, bicuspid aortic valve, dilatation or aneurysm of the ascending aorta and more rarely aortic sinus aneurysm. ${ }^{2}$ These defects can be found separately or in combinations of two or more. ${ }^{2,4}$ Coarctation, bicuspid aortic valve and aneurysm of the ascending aorta have been described as independent risk factors for dissection., ${ }^{2,5}$ Hypertension, which is found with high prevalence in Turner syndrome even in the absence of coarctation, is another risk factor for dissection. ${ }^{2}$ However, this serious complication may occur even though all the above risk factors, which, cause high haemodynamic stress on the aortic wall, are absent. ${ }^{3}$ The fact could be explained as a consequence of the presence of a common structural abnormality of the connective tissue (showing elastic fibre disruption), with variable expression in Turner syndrome, described as 'cystic medial necrosis of the aorta'.,3 In our patient the cystic structures of the aortic wall are probably an expression of the same defect complicated by a mural infection probably after disruption of the intima. 

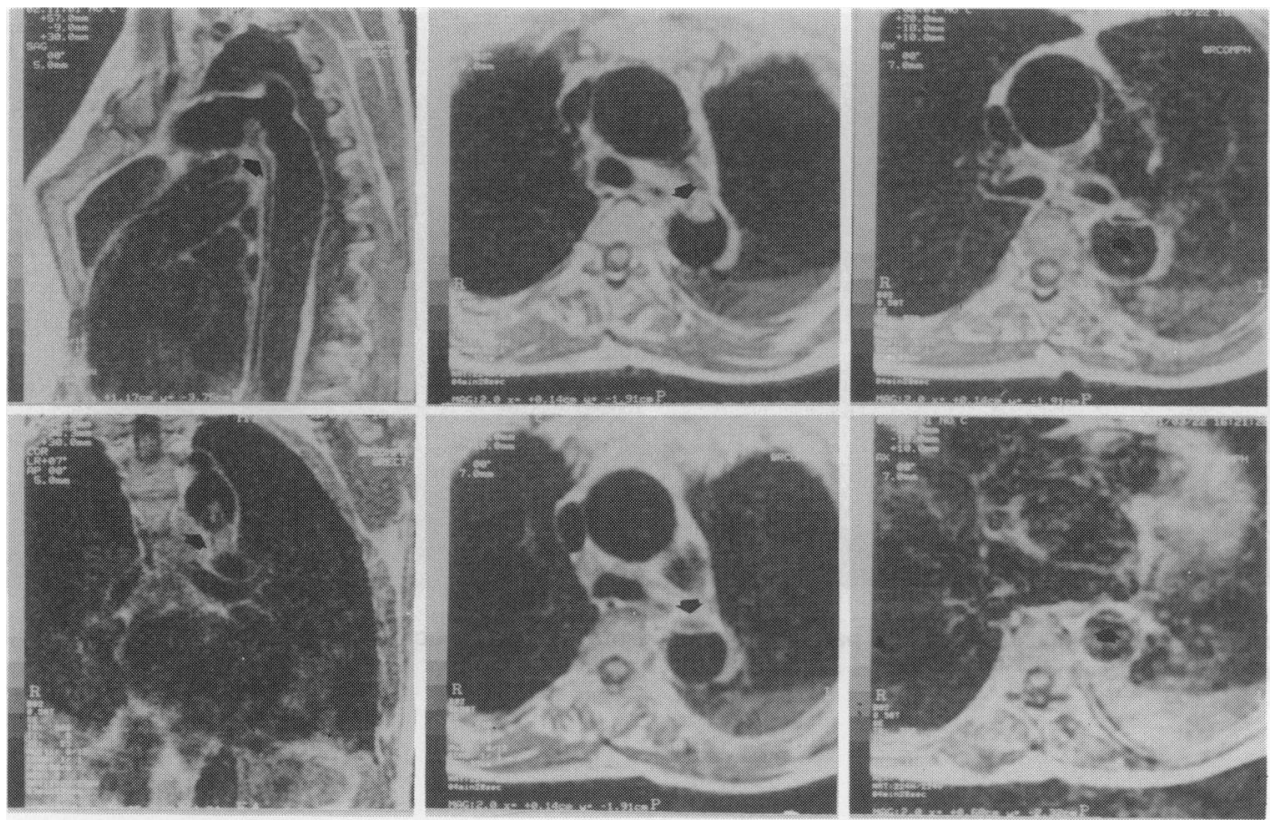

Figure 3 MRI of the patient showing the thickening of the wall of the aortic arch with an intraluminal structure and cystic structures of the descending aorta.

\section{References}

1. Swapp, G.H., Johnston, A.W., Watt, J.L., Couzin, D.A. \& Stephen, G.S. A fertile woman with non-mosaic Turner's syndrome. Case report and review of the literature. Br J Obstet Gynaecol 1989, 96: 876-880.

2. Subramaniam, P.N. Turner's syndrome and cardiovascular anomalies: a case report and review of the literature. Am J Med Sci 1989, 297: 260-262.

3. Price, W.H. \& Wilson, J. Dissection of the aorta in Turner's syndrome. J Med Genet 1983, 20: 61-63.

4. Allen, D.B., Hendricks, S.A. \& Levy, J.M. Aortic dilation in Turner syndrome. J Pediatr 1986, 109: 302-305.

5. Slater, D.N., Grundman, M.J. \& Mitchell, L. Turner's syndrome associated with bicuspid aortic stenosis and dissecting aortic aneurysm. Postgrad Med J 1982, 58: 436-438.
6. Kaufman, S. Vaginal bleeding in gonadal dysgenesis. Pediatrics 1966, 37: 26-33.

7. McDonough, P.G., Byrd, J.R. \& Freedman, M.A. Gonadal dysgenesis with ovarian function. Obstet Gynecol 1971, 37: 868-872.

8. Shokeir, M.H.K. Pregnancy in five women with $45, \mathrm{XO} / 46, \mathrm{XX}$ and $45, \mathrm{XO} / 47, \mathrm{XXX}$ gonadal dysgenesis. In: Summit, D. \& Bergsma, R.L. (eds) Sex Differentiation and Chromosomal Abnormalities. Alan Liss, Inc., New York, 1978, pp. 171-184. 